\title{
Hypersensitivity Pneumonitis High-resolution Computed Tomography Findings, and Their Correlation with the Etiology and the Disease Duration
}

\author{
Farzaneh Shobeirian', Payam Mehrian², Abtin Doroudinia ${ }^{3}$ \\ ${ }^{1}$ Razi Clinical Research Development Unit, Razi Hospital, Guilan University \\ of Medical Sciences, Rasht, Iran; \\ ${ }^{2}$ Telemedicine Research Center (TRC), National Research Institute of Tuberculosis \\ and Lung Diseases (NRITLD), Shahid Beheshti University of Medical Sciences, \\ Tehran, Iran; \\ ${ }^{3}$ Chronic Respiratory Diseases Center, National Research Institute of Tuberculosis \\ and Lung Diseases (NRITLD), Shahid Beheshti University of Medical Sciences, \\ Tehran, Iran
}

Received February 24, 2020; Accepted September 14, 2020.

Key words: Hypersensitivity pneumonitis - Computed tomography - Etiology

Abstract: Hypersensitivity pneumonitis (HP) is an immune-mediated diffuse parenchymal lung disease induced by inhaled antigens. High-resolution computed tomography (HRCT) is widely used in the diagnosis and follow-up of patients and determining the progression and prognosis of the disease. In this retrospective study, 45 consecutive patients with the final diagnosis of HP, seen at a large tertiary care center during a period of 4 years, were included and their HRCT findings were evaluated. The most common HRCT findings were ground glass opacity and reticulation. Some HRCT patterns were detected more severely in bird fanciers in comparison with other etiologies. There is no "gold standard" for the diagnosis of HP. HRCT findings play an important role in hypersensitivity pneumonitis diagnosis and CT scan also help to define the severity of hypersensitivity pneumonitis injuries. In our study, reticulation and ground glass opacity were the most common findings in HRCT of patients with HP. We also find that patients with avian contacts had a significantly higher rate of fibrosis.

Mailing Address: Dr. Payam Mehrian, Department of Radiology, Masih Daneshvari Hospital, Tehran, Iran; Mobile Phone: +989 121548 869; e-mail: drpayam.mehrian@gmail.com 


\section{Introduction}

Hypersensitivity pneumonitis (HP), also known as extrinsic allergic alveolitis, is an immune-mediated diffuse parenchymal lung disease, induced by repeated inhalation of an antigen in a sensitized host (Hanak et al., 2007; Mooney et al., 2013). The number of known antigens is extensive and increasing and those causing farmer's lung and pigeon breeder's/birds fancier's diseases are the most common (Hanak et al., 2007; Rosenman, 2009; Franks and Galvin, 2010). HP is clinically classified as acute, subacute and chronic. The most common forms of the disease are acute and subacute with only a low percentage progress to chronic forms (Walsh et al., 2012). Patients with chronic HP have radiologic and histopathologic evidence of fibrosis (Cormier et al., 2000). In all forms of HP, there is no "gold standard" test or approach. One widely used criteria include: 1) symptoms compatible with HP, 2) evidence of an appropriate antigen exposure by either history or an antibody testing result, 3) presence of episodic respiratory symptoms that correlates with recurrent antigen exposure, 4) imaging findings compatible with HP, 5) a lymphocytosis on BAL, and 6) histopathologic features compatible with HP (Lacasse et al., 2012; Mason et al., 2016; Pereira et al., 2016; Vasakova et al., 2017; Dias et al., 2018). The radiographic chest $X$-ray has always been important in diagnosis, however, the recently developed high resolution computed tomography (HRCT) has become a highly informative diagnostic parameter, which is more sensitive and the normal HRCT is a strong evidence against the diagnosis of active HP (Cormier et al., 2000; Franks and Galvin, 2010). HRCT is widely used in the follow-up of patients, determining the treatment responses and prognosis of the disease (Walsh et al., 2012; Dias et al., 2018). The outcome of HP is variable and depends on clinical and radiological presentation (Vasakova et al., 2017). It seems that HRCT findings are sufficient for the diagnosis of HP in the setting of antigen exposure and exclusion of other differential diagnoses. The characteristic HRCT features of HP are ground glass opacities, centrilobular nodules, and mosaic attenuation. Other findings include consolidation, reticulation, fibrosis, emphysema, and cysts. These findings have been described mostly in middle and upper lung zones and HP classically spares the pulmonary bases. Ground glass opacities represent active inflammation or fibrosis and are predominant in acute and subacute forms. Centrilobular nodules are mostly detected in subacute form. However, they can also be associated with fibrosis in chronic forms which mainly have ground glass opacity. Mosaic attenuation is described in chronic HP and is more apparent in expiratory scans. Reticulation is the most frequent finding in chronic HP. Consolidation is not the main abnormality in HP HRCT and may represent areas of organizing pneumonia or superimposed infections or acute exacerbation (Adler et al., 1992; Cormier et al., 2000; Silva et al., 2007; Rosenman, 2009; Franks and Galvin, 2010; Akira and Suganuma, 2014; Lynch, 2016; Pereira et al., 2016; Vasakova et al., 2017; Dias et al., 2018). 


\section{Material and Methods}

Study population

In this retrospective study, 45 consecutive patients with the final diagnosis of HP seen at our tertiary lung center, were included. HP diagnosis was based on clinical history including chronic respiratory symptoms and history of antigen exposure, abnormal pulmonary function tests, and radiologic findings and compatible HRCT reviewed by thoracic radiologists, exclusion of other mimic diseases, bronchoalveolar lavage (BAL), and lung biopsy. Data from all patients were collected by questionnaires and physician review. If there were any further question or unclear history, patients were contacted again. All the patients signed the written consent forms. Patients' demographics, occupation, history of tobacco, antigen exposure, lab tests, pulmonary function tests, and HRCT findings reviewed by thoracic radiologists were recorded. Antigens exposure determined by clinician were classified to plants antigens, birds related antigens, pets, chemical materials or unknown origin.

\section{Radiographic assessment}

HRCT was performed using 16-slice multi-detector CT scanner (SOMATOM Definition Flash, Seimens Healthcare, Forchheim, Germany). All patients were examined with the supine position in a suspended deep inspiration from lung apices to lung bases with standard acquisition $200 \mathrm{~mA}, 120 \mathrm{kVp}, 1.25 \mathrm{~mm}$ collimation and $10 \mathrm{~mm}$ intervals. No intravenous contrast was used in any of the included HRCT studies. Ground glass opacity was defined as increased lung opacity without obscuring pulmonary architecture or pulmonary vessels (Austin et al., 1996). Centrilobular nodules were defined as small round opacities measuring less than $7 \mathrm{~mm}$ in the center of pulmonary secondary lobules (Austin et al., 1996; Cormier et al., 2000). Mosaic attenuation was defined as a patchwork of areas with different attenuation (Figure 1). Consolidation was defined as an area of increased parenchymal opacity obscuring vessels and airway margins (Austin et al., 1996). Reticulation was considered as linear opacities forming a mesh appearance (Austin et al., 1996). Emphysema and cysts were defined as areas of low attenuation without and with thin wall respectively (Austin et al., 1996; Cormier et al., 2000). Honeycombing was defined as a subpleural cluster of well-defined cystic airspaces of different sizes (Figure 2) (Austin et al., 1996). Traction bronchiectasis was also defined as irregular bronchial dilation around or within abnormal parenchyma (Figure 3) (Austin et al., 1996; Cormier et al., 2000; Tateishi et al., 2011). Fibrosis was defined as the presence of honeycombing, linear irregular opacities, or traction bronchiectasis (Hanak et al., 2008). The presence of HRCT findings was determined by two thoracic radiologists separately and the distribution was also specified in lung zones.

HRCT images were reviewed by two thoracic radiologists and the results were achieved by consensus. 
136) Prague Medical Report / Vol. 121 (2020) No. 3, p. 133-141
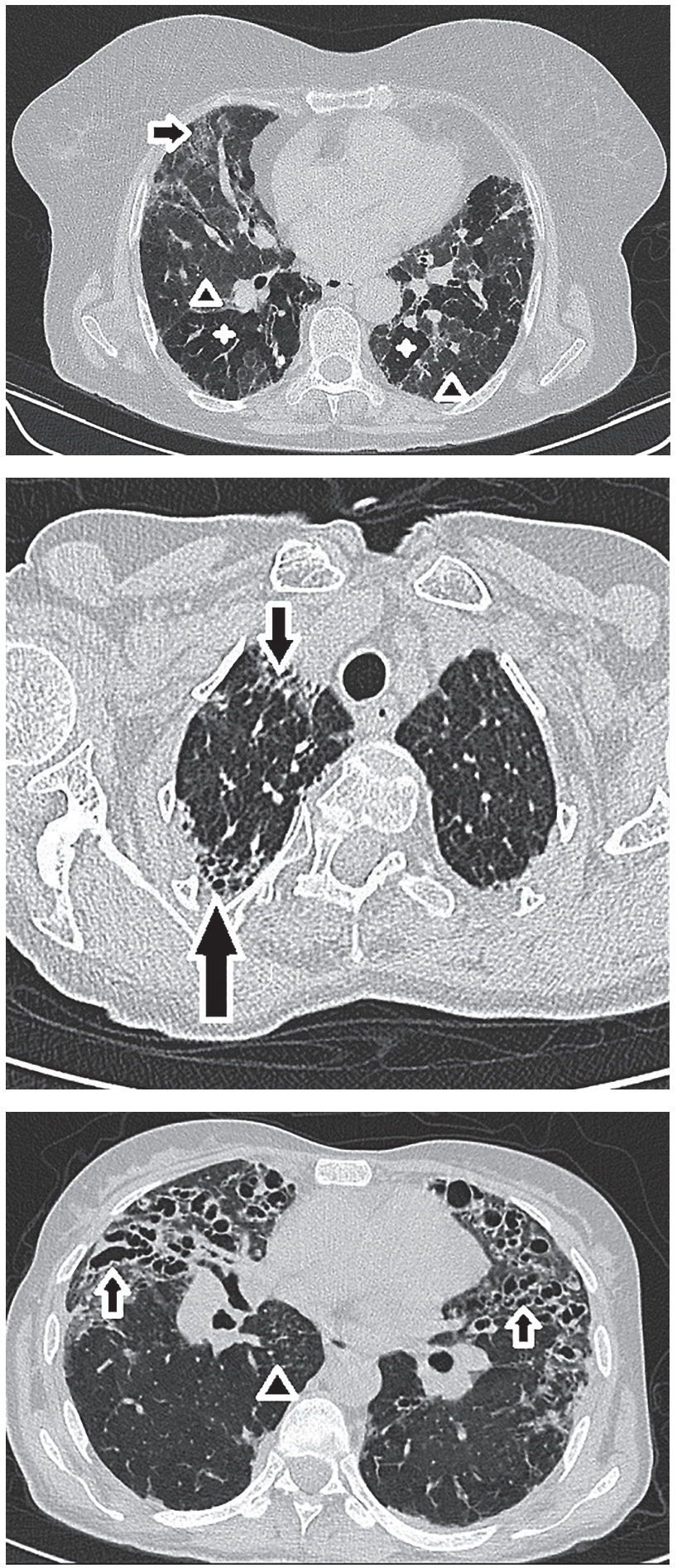

Figure 1 - Hypersensitivity

pneumonitis. Axial high-resolution computed tomography demonstrates mosaic attenuation, "head cheese sign". Areas with normal parenchyma (arrowhead), areas of air trapping (four-point star), and ground glass opacity (arrow).

Figure 2 - Hypersensitivity pneumonitis. Axial high-resolution computed tomography demonstrates honeycombing.

Figure 3 - Hypersensitivity pneumonitis. Axial high-resolution computed tomography demonstrates traction bronchiectasis (arrow), centrilobular nodules (arrowhead). 
Statistical analysis

Analyses were performed with Statistical Package for Social Sciences (SPSS) software version 19. Categorical variables were described by percentages and were compared by Chi-square or Fisher's exact tests as appropriate. Continuous variables were described by means and standard deviation and were evaluated by $t$-test. A p-value $<0.05$ was considered as statistically significant. Lungs were considered as six zones (upper, middle and lower zones on each side).

\section{Results}

In this study, 45 patients (23 males, 22 females) with hypersensitivity pneumonitis diagnosis were enrolled. The average age of patients was $53.24 \pm 18.69$ (range 20-86) years old. The mean time from onset of clinical symptoms was $7.47 \pm 6.18$ (range 1-28) years. The prevalence of HRCT findings was $93.3 \%$ for reticulation and ground glass opacity, $75.6 \%$ for fibrosis, and $71.1 \%$ for air trapping and traction bronchiectasis as classified in Table 1. The predominant findings on HRCT were reticulation and ground glass opacity. Reticulation was mostly diffuse in lungs in $76.1 \%$. Reticulation was diffuse bilateral in $50 \%$ of patients, in $6.2 \%$ of patients ground glass opacity was bilateral in lower lobes and in $6.2 \%$ was bilateral in the upper lobes, the other patients did not have specific distribution. There was no significant difference in HRCT features between males and females. In comparison between patients less than 50 years of age and patients with older age than 50 years old, there were significant differences in air trapping and traction bronchiectasis. Air trapping was more common in patients older age than 50 years

\section{Table 1 - High-resolution computed tomography findings prevalence in $\mathbf{4 5}$ patients with hypersensitivity pneumonitis}

\begin{tabular}{lcc}
\hline HRCT feature & Cases & Prevalence (\%) \\
\hline Reticulation & 42 & 93.3 \\
Ground glass opacity & 42 & 93.3 \\
Fibrosis & 34 & 75.6 \\
Air trapping & 32 & 71.1 \\
Traction bronchiectasis & 32 & 71.1 \\
Ground glass nodules & 27 & 60.0 \\
Solid nodules & 6 & 13.3 \\
Consolidation & 28 & 62.2 \\
Emphysema/cysts & 17 & 37.8 \\
Honeycombing & 16 & 35.6 \\
Mosaic attenuation & 26 & 57.8 \\
Costophrenic angle sparing & 22 & 48.9 \\
Pleural reaction & 6 & 13.3 \\
Lymphadenopathy & 20 & 44.4 \\
\hline
\end{tabular}

HRCT - high-resolution computed tomography 


\section{Table 2 - Etiologic various exposure in $\mathbf{4 5}$ patients with hypersensitivity pneumonitis}

\begin{tabular}{lcc}
\hline Etiology & Cases & Prevalence (\%) \\
\hline Unknown & 13 & 28.9 \\
Molded and agriculture-related antigen & 11 & 24.4 \\
Birds & 7 & 15.6 \\
Other pets & 2 & 4.4 \\
Various chemicals & 12 & 26.7 \\
\hline
\end{tabular}

old $(95.7 \%$ vs. $45.5 \%$, p-value $<0.001)$. Traction bronchiectasis was also more common in patients older age than 50 years old ( $91.3 \%$ vs. $50 \%$, p-value $=0.002$ ). Of these 45 patients, there was no known etiology in $28.9 \%$, and so on $26.7 \%$ had contacts with agriculture-related antigens, $15.6 \%$ had contacts with birds, $4.4 \%$ were exposed to the other pets' antigens, and $28.9 \%$ were exposed to different types of chemicals (Table 2). In our study, patients with avian contacts had a significantly higher rate of fibrosis in comparison with other etiologies $(81.6 \%$ vs. $42.9 \%$, $\mathrm{p}$-value $=0.028$ ). There was significant higher rate of traction bronchiectasis and honeycombing in patients with avian contacts compared to other etiologies (78.9\% vs. $28.6 \%, p$-value $=0.015$ and $42.1 \%$ vs. $0 \%, p$-value $=0.032$, respectively). There were no significant differences in HRCT patterns of other etiologies. In comparison of patients with disease duration more than 5 years and patients with duration less than 5 years, there was significant higher rate of air trapping and emphysema/cysts in patients with disease duration more than 5 years $(85.7 \%$ vs. $58.3 \%$, $p$-value $=0.043$, and $52.4 \%$ vs. $25 \%$, $p$-value $=0.05$ ). Fibrosis had a significantly higher prevalence in patients with disease duration more than 5 years $(95.2 \%$ vs. $58.3 \%$, $p$-value $=0.004)$. There was a higher rate of traction bronchiectasis in patients with disease duration more than 5 years compared to patients with shorter duration of disease.

\section{Discussion}

In this study, we describe HRCT findings in 45 patients with a final diagnosis of HP. Dias et al. (2018) reviewed the essential features of CT in hypersensitivity pneumonitis and discussed HP as a complex disease with reticular opacities, traction bronchiectasis, and architectural distortion as main CT findings. They also mentioned that in advanced stages, HP may resemble nonspecific interstitial pneumonia (NSIP) or usual interstitial pneumonia (UIP) but evidence of small airways involvement such as centrilobular ground-glass opacities, peribronchovascular involvement, upper lobe predominance and mosaic pattern can help the diagnosis (Dias et al., 2018). In our study, the predominant findings on HRCT were reticulation and ground glass opacity and our study confirmed the previous studies that ground glass opacity and findings of fibrosis, namely 
reticulation, architectural distortion, and traction bronchiectasis with or without honeycomb change are most common (Cormier et al., 2000; Tateishi et al., 2011; Walsh et al., 2012; Vasakova et al., 2017). Vasakova et al. (2017) have described emphysema in patients with HP. Cormier et al. also confirm previous reports that emphysema is a long-term outcome in patients with farmer's disease (ErkinjunttiPekkanen et al., 1998; Cormier et al., 2000). In our study, patients with disease duration more than 5 years had a higher rate of emphysema and air trapping. Air trapping was more common in patients with older age than 50 years old.

Walsh et al. (2012) implied that HRCT is increasingly used to assess chronic hypersensitivity pneumonitis. They also suggested that HRCT patterns are superior to pulmonary function tests for mortality prediction and extensive traction bronchiectasis strongly predicts poor survival in chronic hypersensitivity pneumonitis (Walsh et al., 2012). Some other studies also mentioned that fibrosis extent could be a high confidential prognostic factor (Hanak et al., 2008; Mooney et al., 2013; Pereira et al., 2016). In our study, fibrosis had a significantly higher prevalence in patients with disease duration more than 5 years and there was a higher rate of traction bronchiectasis in patients with disease duration more than 5 years compared to patients with shorter duration of disease.

Hanak et al. (2007) notified that avian antigens were the most common etiology of HP (34\%) in their study and in $25 \%$ of the patients inciting antigen could not be identified. Inciting antigens are different in various geographic regions depending on climate, culture, socioeconomic status, and occupational factors. In England, the most common etiology of HP has been reported avian antigens, versus molds in Japan (Hendrick et al., 1978; Bourke et al., 2001; Hanak et al., 2007). In our study, the most common antigens causing HP were molded and agriculture-related antigens (24.4\%), birds' antigens were responsible in $15.6 \%$ of patients, and $28.9 \%$ had unknown etiology.

In our study, patients with avian contacts had a significantly higher rate of fibrosis in comparison with other etiologies. There was a significantly higher rate of traction bronchiectasis and honeycombing in patients with avian contacts compared to other etiologies. There were no significant differences in HRCT patterns of other etiologies. This may suggest that HP with avian contacts may have more severe HRCT patterns and subsequently poorer prognosis which needs further evaluation and studies to be proved.

\section{Conclusion}

HRCT findings are an important part of hypersensitivity pneumonitis diagnosis. $\mathrm{CT}$ can also help to define the severity of hypersensitivity pneumonitis injuries. In this study, we found that reticulation and ground glass opacity were the most common findings in HRCT of patients with HP. It was also noted that air trapping, emphysema, and fibrosis had a higher rate in patients with disease duration more than 5 years. Patients with avian contacts had a significantly higher rate of fibrosis in 
comparison with other etiologies but there were no significant differences in HRCT patterns of other etiologies.

\section{References}

Adler, B., Padley, S., Müller, N., Remy-Jardin, M., Remy, J. (1992) Chronic hypersensitivity pneumonitis: High-resolution CT and radiographic features in 16 patients. Radiology 185(1), 91-95.

Akira, M., Suganuma, N. (2014) Acute and subacute chemical-induced lung injuries: HRCT findings. Eur. J. Radiol. 83(8), 1461-1469.

Austin, J., Müller, N., Friedman, P. J., Hansell, D. M., Naidich, D. P., Remy-Jardin, M., Webb, W. R., Zerhouni, E. A. (1996) Glossary of terms for CT of the lungs: Recommendations of the Nomenclature Committee of the Fleischner Society. Radiology 200(2), 327-331.

Bourke, S., Dalphin, J., Boyd, G., McSharry, C., Baldwin, C., Calvert, J. (2001) Hypersensitivity pneumonitis: current concepts. Eur. Respir. J. 18, 81s-92s (Suppl. 32).

Cormier, Y., Brown, M., Worthy, S., Racine, G., Muller, N. (2000) High-resolution computed tomographic characteristics in acute farmer's lung and in its follow-up. Eur. Respir. J. 16(1), 56-60.

Dias, O. M., Baldi, B. G., Pennati, F., Aliverti, A., Chate, R. C., Sawamura, M. V. Y., Carvalho, C. R. R., de Albuquerque, A. L. P. (2018) Computed tomography in hypersensitivity pneumonitis: Main findings, differential diagnosis and pitfalls. Expert Rev. Respir. Med. 12(1), 5-13.

Erkinjuntti-Pekkanen, R., Rytkonen, H., Kokkarinen, J. I., Tukiainen, H. O., Partanen, K., Terho, E. O. (1998) Long-term risk of emphysema in patients with farmer's lung and matched control farmers. Expert Rev. Respir. Med. 158(2), 662-665.

Franks, T. J., Galvin, J. R. (2010) Hypersensitivity pneumonitis: Essential radiologic and pathologic findings. Surg. Pathol. Clin. 3(1), 187-198.

Hanak, V., Golbin, J. M., Ryu, J. H. (2007) Causes and presenting features in 85 consecutive patients with hypersensitivity pneumonitis. Paper presented at the Mayo Clinic Proceedings.

Hanak, V., Golbin, J. M., Hartman, T. E., Ryu, J. H. (2008) High-resolution CT findings of parenchymal fibrosis correlate with prognosis in hypersensitivity pneumonitis. Chest 134(1), 133-138.

Hendrick, D., Faux, J. A., Marshall, R. (1978) Budgerigar-fancier's lung: The commonest variety of allergic alveolitis in Britain. Br. Med. J. 2(6130), 81-84.

Lacasse, Y., Girard, M., Cormier, Y. (2012) Recent advances in hypersensitivity pneumonitis. Chest 142(1), 208-217.

Lynch, J. P. (2016) Interstitial Pulmonary and Bronchiolar Disorders. CRC Press, Boca Raton.

Mason, R. J., Slutsky, A., Murray, J. F., Nadel, J. A., Law, D., Gotway, M. (2016) Murray and Nadel's Textbook of Respiratory Medicine, 2-Volume Set, $6^{\text {th }}$ Edition. Elsevier, Amsterdam.

Mooney, J. J., Elicker, B. M., Urbania, T. H., Agarwal, M. R., Ryerson, C. J., Nguyen, M. L. T., Woodruff, P. G., Jones, K. D., Collard, H. R., King, T. E. Jr., Koth, L. L. (2013) Radiographic fibrosis score predicts survival in hypersensitivity pneumonitis. Chest 144(2), 586-592.

Pereira, C. A., Gimenez, A., Kuranishi, L., Storrer, K. (2016) Chronic hypersensitivity pneumonitis. J. Asthma Allergy $9,171$.

Rosenman, K. D. (2009) Asthma, hypersensitivity pneumonitis and other respiratory diseases caused by metalworking fluids. Curr. Opin. Allergy Clin. Immunol. 9(2), 97-102.

Silva, C. I. S., Churg, A., Müller, N. L. (2007) Hypersensitivity pneumonitis: Spectrum of high-resolution CT and pathologic findings. AJR Am. J. Roentgenol. 188(2), 334-344.

Tateishi, T., Ohtani, Y., Takemura, T., Akashi, T., Miyazaki, Y., Inase, N., Yoshizawa, Y. (2011) Serial high-resolution computed tomography findings of acute and chronic hypersensitivity pneumonitis induced by avian antigen. J. Comput. Assist. Tomogr. 35(2), 272-279. 
Vasakova, M., Morell, F., Walsh, S., Leslie, K., Raghu, G. (2017) Hypersensitivity pneumonitis: Perspectives in diagnosis and management. Am. J. Respir. Crit. Care Med. 196(6), 680-689.

Walsh, S. L., Sverzellati, N., Devaraj, A., Wells, A. U., Hansell, D. M. (2012) Chronic hypersensitivity pneumonitis: High resolution computed tomography patterns and pulmonary function indices as prognostic determinants. Eur. Radiol. 22(8), 1672-1679. 